\title{
Kaiso depletion attenuates the growth and survival of triple negative breast cancer cells
}

\author{
Blessing I Bassey-Archibong1, Lyndsay GA Rayner', Shawn M Hercules ${ }^{1}$, Craig W Aarts ${ }^{2}$, Anna Dvorkin-Gheva ${ }^{2,3}$, Jonathan L Bramson², \\ John A Hassell ${ }^{3}$ and Juliet M Daniel ${ }^{*, 1}$
}

Triple negative breast cancers (TNBC) are highly aggressive and lack specific targeted therapies. Recent studies have reported high expression of the transcription factor Kaiso in triple negative tumors, and this correlates with their increased aggressiveness. However, little is known about the clinical relevance of Kaiso in the growth and survival of TNBCs. Herein, we report that Kaiso depletion attenuates TNBC cell proliferation, and delays tumor onset in mice xenografted with the aggressive MDA-231 breast tumor cells. We further demonstrate that Kaiso depletion attenuates the survival of TNBC cells and increases their propensity for apoptotic-mediated cell death. Notably, Kaiso depletion downregulates BRCA1 expression in TNBC cells expressing mutant-p53 and we found that high Kaiso and BRCA1 expression correlates with a poor overall survival in breast cancer patients. Collectively, our findings reveal a role for Kaiso in the proliferation and survival of TNBC cells, and suggest a relevant role for Kaiso in the prognosis and treatment of TNBCs.

Cell Death and Disease (2017) 8, e2689; doi:10.1038/cddis.2017.92; published online 23 March 2017

Triple negative breast cancers (TNBC) represent a heterogeneous subtype of breast tumors that generally lack expression of estrogen receptor (ER), progesterone receptor (PR) and the human epidermal growth factor receptor $2 .{ }^{1}$ TNBCs are highly proliferative and have a high rate of recurrence compared to other breast cancer $(\mathrm{BCa})$ subtypes. ${ }^{2}$ Currently, there are no specific targeted therapies for the management of TNBC, hence treatment is limited to radio- and chemotherapy. Although TNBCs initially respond to chemotherapy, many patients relapse and this contributes to a shortened overall survival for affected patients. ${ }^{3}$

Various proteins have been implicated in the survival and chemo-resistant nature of TNBC. Two of the most understood are the tumor suppressors BRCA1 and p53. ${ }^{4-6}$ BRCA1 is mutated in $\sim 45 \%$ of familial $\mathrm{BCa}^{7}$ and a high proportion of sporadic $\mathrm{BCa}$, especially of the TNBC subtype. ${ }^{8,9}$ However, some TNBCs retain the expression of wild-type (wt) BRCA1 (which plays a role in DNA repair) and this has been associated with their resistance to chemotherapeutic drugs such as Cisplatin. ${ }^{10}$ Similarly, p53 is mutated in $\sim 30 \%$ of $\mathrm{BCa}^{11}$ with a higher frequency observed in TNBCs, reviewed in Walerych et al. ${ }^{12}$ The inability of mutant p53 to bind and activate the expression of canonical p53 target genes such as the pro-apoptotic genes Noxa, Bax and Puma is believed to contribute to the chemo-resistance and survival of $\mathrm{BCa} .{ }^{13-18}$

Several recent studies have implicated increased nuclear expression of the transcription factor Kaiso in the aggressiveness of certain tumors including basal/triple negative tumors. ${ }^{19,20}$ Kaiso is a POZ-ZF transcription factor that was first identified as a binding partner of the E-cadherin catenin cofactor p120-catenin $\left(\mathrm{p} 120^{\mathrm{ctn}}\right) .{ }^{21}$ In the absence or downregulation of E-cadherin, $\mathrm{p} 120^{\mathrm{ctn}}$ is able to translocate to the nucleus ${ }^{22,23}$ where it can bind and inhibit Kaiso's transcriptional activities. ${ }^{23-25}$ Although high Kaiso expression is associated with TNBC aggressiveness, ${ }^{19,26}$ Kaiso's specific role in the growth and survival of TNBCs remains unknown. Interestingly, roles for Kaiso in cell growth (proliferation) and survival (decreased apoptosis) have been demonstrated in several cell types. For instance, Kaiso depletion results in decreased Cyclin D1, reduced proliferation and increased apoptosis of cervical cancer (HeLa) cells, but decreased apoptosis of human embryonic kidney (HEK293) cells. ${ }^{27}$ Similarly, loss of Kaiso decreased prostate tumor cell proliferation $^{20}$ and delayed the onset of intestinal polyp formation in $\mathrm{Apc}^{\mathrm{Min+}}$ mice. ${ }^{28}$ Finally, loss of Kaiso-mediated transcriptional repression is associated with increased anchorage-independent cell growth of mouse lobular $\mathrm{BCa}$ cells. ${ }^{23}$ Collectively, these studies suggest context-dependent roles for Kaiso in cell proliferation and apoptosis.

Herein, we report that Kaiso depletion attenuates the proliferative ability of TNBC cells, reduces the anchorageindependent growth of MDA-231 cells and delays the tumor onset of MDA-231 xenografts. We also show that Kaiso depletion increases the apoptosis of TNBC cells. More importantly, we report for the first time that silencing Kaiso results in the downregulation of BRCA1 in mutant-p53expressing TNBC cells. Together, our findings suggest that high Kaiso expression promotes the growth and survival of TNBCs and raise the possibility that Kaiso may be a useful biomarker for the prognosis and treatment of a subset of TNBCs.

\footnotetext{
${ }^{1}$ Department of Biology, McMaster University, Hamilton, Ontario L8S 4K1, Canada; ${ }^{2}$ Department of Pathology and Molecular Medicine, McMaster University, Hamilton, Ontario L8S 4K1, Canada and ${ }^{3}$ Department of Biochemistry and Biomedical Sciences, McMaster University, Hamilton, Ontario L8S 4K1, Canada ${ }^{*}$ Corresponding author: JM Daniel, Department of Biology, McMaster University, LSB-331, 1280 Main Street West, Hamilton, Ontario L8S 4K1, Canada. Tel: +1 9055259140 ext. 23765; Fax: +1 905522 6066; E-mail: danielj@mcmaster.ca Received 12.10.16; revised 12.1.17; accepted 06.2.17; Edited by R Johnstone
} 

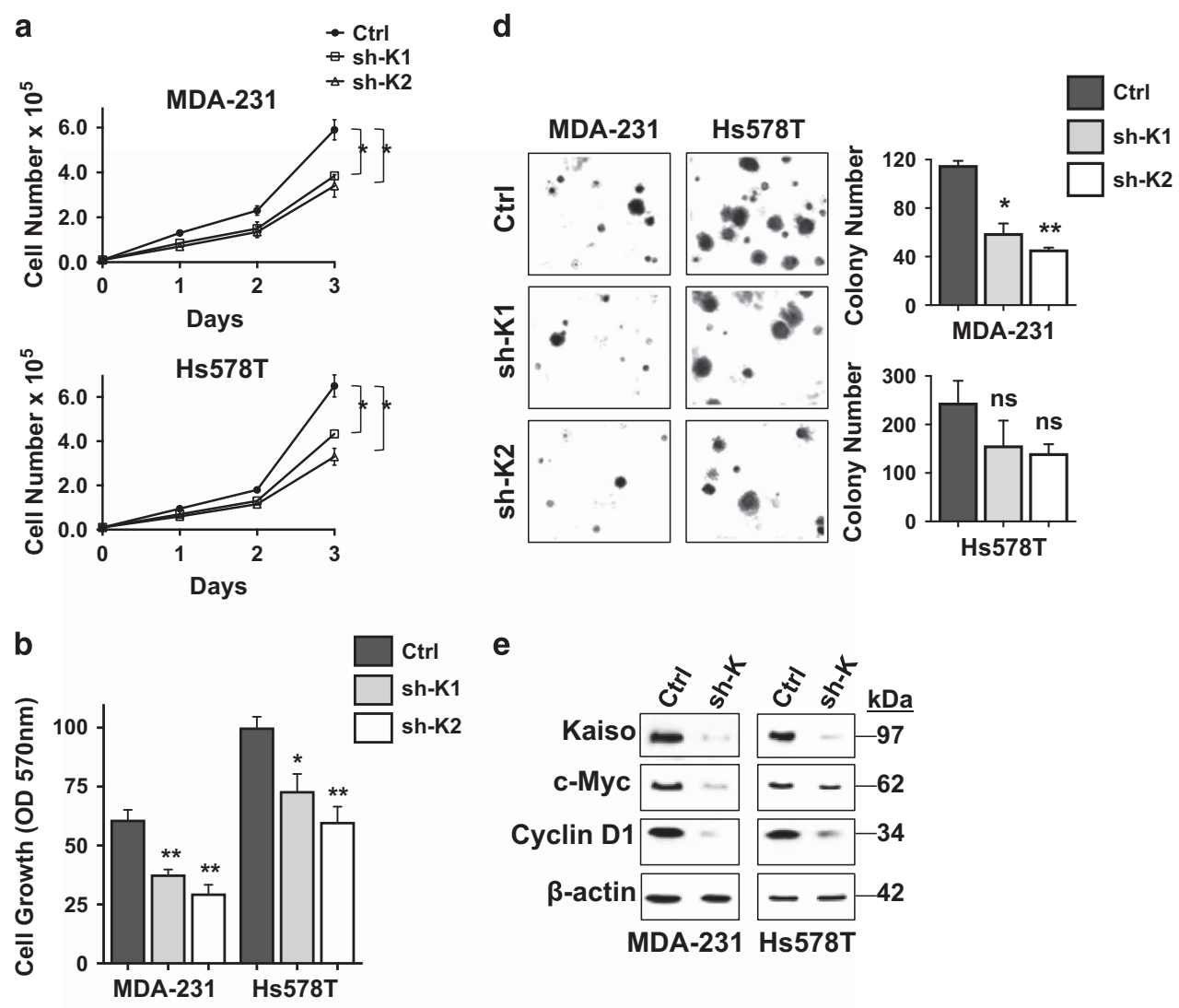

C
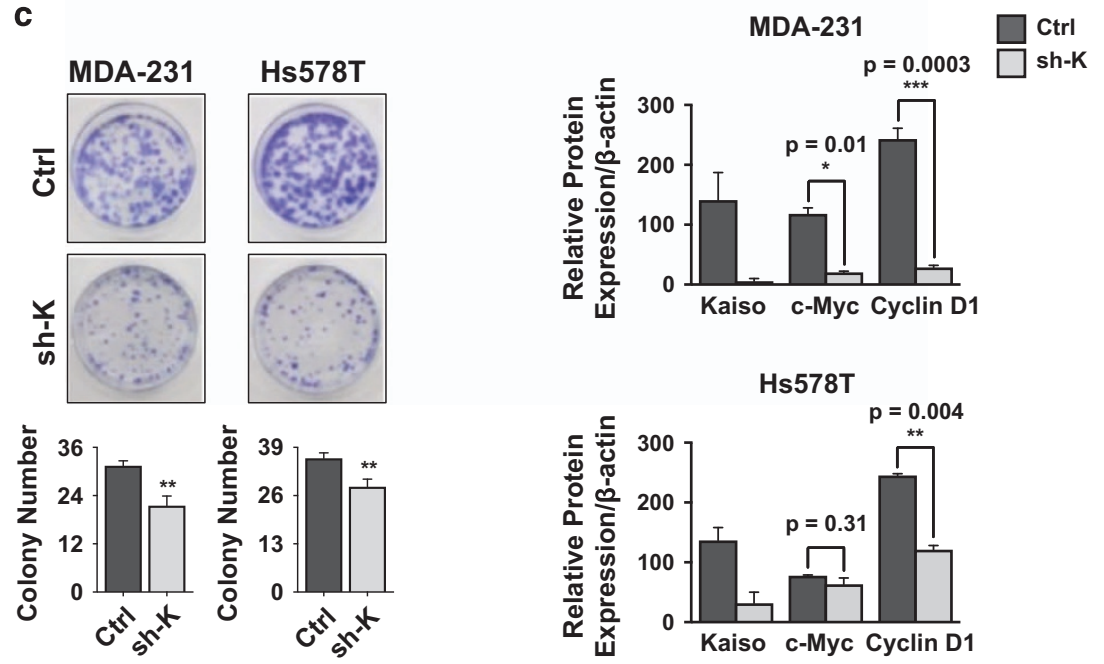

Figure 1 Kaiso depletion attenuates the proliferation of TNBC cells. Growth curve (a), MTT assays (b) and colony formation assays (c) were conducted on Ctrl and Kaisodepleted (sh-K1 and sh-K2, hereafter referred to as sh-K) cells, and reveal that Kaiso depletion inhibits proliferation of MDA-231 and Hs578T cells. (d) Soft agar assays were also conducted on Ctrl, sh-K1 and sh-K2 MDA-231 and Hs578T cells and revealed that Kaiso-depletion diminished anchorage-independent growth of MDA-231 but not Hs578T cells. (e) Kaiso depletion resulted in decreased c-Myc and cyclin D1 expression in MDA-231 and Hs578T cells as detected by IB analysis and densitometry analysis. The reduction in c-Myc levels in response to Kaiso depletion was more significant in MDA-231 compared to Hs578T cells. GraphPad Prism software was used to generate graphs and for all statistical calculations. Data shown are representative of three independent experiments. ${ }^{\star} P<0.05,{ }^{\star \star} P<0.01$ and ${ }^{\star \star \star} P<0.001$

\section{Results}

Kaiso depletion inhibits TNBC cell proliferation. Recently, we reported that Kaiso is highly expressed in triple negative tumors and correlates with the metastatic propensity of TNBC cells. ${ }^{19,26}$ To ascertain whether Kaiso is also involved in TNBC cell growth and survival, we performed cell viability assays (direct cell counts, MTT and colony formation assays) on control (Ctrl) and Kaiso-depleted (sh-K1 and sh-K2, hereafter referred to as sh-K) TNBC cell lines (MDA-231 and Hs578T), see Bassey-Archibong et al. ${ }^{26}$ Notably, silencing Kaiso significantly inhibited the proliferation 
of MDA-231 and Hs578T cells in all assays performed (Figures $1 \mathrm{a}-\mathrm{C}$ ). We also conducted soft agar assays on Ctrl and Kaiso-depleted MDA-231 and Hs578T cells, and found that Kaiso-depletion mitigates the anchorage independence of MDA-231 but not Hs578T cells (Figure 1d). As previous studies had implicated Kaiso in the regulation of the proproliferation genes $c-m y c$ and cyclin $D 1,{ }^{24,29,30}$ we examined the expression of these proteins in Kaiso-depleted TNBC cells. We found that Kaiso-depleted MDA-231 and Hs578T cells expressed less c-Myc and Cyclin D1 than control cells (Figure 1e and Supplementary Figure 1A), which further supported a role for Kaiso in cell proliferation.

Kaiso depletion results in delayed tumor onset of MDA-231 xenografts. To ascertain whether the in vitro effect of Kaiso depletion on TNBC cell proliferation would be sustained in vivo, we performed mouse xenograft studies with the well-characterized TNBC cell line-MDA-231. Equal numbers $\left(4.5 \times 10^{6}\right)$ of Ctrl and Kaiso-depleted (sh-K) MDA-231 cells were injected into the mammary fat pad of immunocompromised mice $(n=5$ for each experimental condition) and allowed to form tumors as previously described. $^{26}$ Interestingly, we observed a significant delay ( $\sim$ weeks) in tumor formation in the Kaiso-depleted xenografts compared to controls which took $\sim 5$ weeks to form visible tumors (Figure 2a). Furthermore, upon tumor formation, the Kaiso-depleted tumors took $\sim 4$ weeks to reach the endpoint size of $3300 \mathrm{~mm}^{3}$ compared to the control tumors which took $\sim 3$ weeks to reach $3300 \mathrm{~mm}^{3}$ (Figure 2a). To determine if the delayed tumor onset observed in the Kaiso-depleted xenografts was due to Kaiso-depletion effects on proliferation, size-matched $\left(\sim 3300 \mathrm{~mm}^{3}\right) \mathrm{Ctrl}$ and sh-K MDA-231 tumor tissues were harvested and examined for the expression of the well-established proliferation markers (Ki-67 and PCNA). Immunohistochemical (IHC) analyses revealed less proliferating cells in the Kaiso-depleted tumor tissues compared to control tissues (Figure $2 \mathrm{~b}$ and see Bassey-Archibong et al. ${ }^{26}$ for IHC analysis of Kaiso expression in the $\mathrm{Ctrl}$ and sh-K tumor tissues). This suggests that the delayed tumorigenesis of the Kaiso-depleted MDA-231 cells may be due to their reduced proliferative capacity. However, the delayed tumor onset may also be due to the reduced colonization of Kaiso-depleted MDA-231 cells, since these cells displayed decreased anchorage-independence in vitro (Figure 1d). Nonetheless, consistent with our in vitro proliferation studies, IHC analysis revealed reduced c-Myc and Cyclin D1 expression in Kaiso-depleted MDA-231 tumors compared to control MDA-231 tumor tissues (Figures 2c and d). Collectively, these findings further support a role for Kaiso in TNBC cell proliferation.

Kaiso depletion induces apoptosis in TNBC cells. As the delay in MDA-231 tumor onset could also have been due to increased apoptosis in Kaiso-depleted cells, we investigated the effect of Kaiso depletion on the expression of the apoptotic/cell-death marker-cleaved Caspase 3 (c-Caspase 3) in MDA-231 tumor tissues. Remarkably, we observed an increased number of c-Caspase 3 stained cells in Kaisodepleted MDA-231 tumors compared to control MDA-231 tumors (Figure 3a). Quantification of the Caspase 3 activity of control and Kaiso-depleted (sh-K1 \& sh-K2) MDA-231 cells in vitro, using the Caspase 3 colorimetric assay, also revealed increased Caspase 3 activity in the Kaiso-depleted (sh-K1 \& sh-K2) MDA-231 cells compared to control cells (Figure $3 b$ ). Similar results were also observed in Kaiso-depleted (sh$\mathrm{K} 1$ \& sh-K2) Hs578T cells compared to their control counterparts (Figure 3b). Further verification of Kaiso depletion effects on apoptosis with the Annexin V-fluorescein isothiocyanate (FITC) staining assay also confirmed that Kaiso depletion resulted in increased apoptosis of MDA-231 and Hs578T cells as evidenced by the elevated number of Annexin V-FITC stained cells in Kaiso-depleted (sh-K) cells compared to controls (Figure $3 \mathrm{c}$ ). Similar results were also obtained in an additional TNBC cell line-MDA-157 (Supplementary Figure 2A). To determine if the increased apoptosis in the TNBC cells was specific to Kaiso depletion, we expressed a sh-resistant murine Kaiso cDNA (mKaiso) in the MDA-231 and Hs578T sh-K cells, and subjected these cells to Annexin V-FITC staining. As observed in Figure 3d, Kaiso re-expression rescued the apoptotic phenotype observed in the Kaiso-depleted (sh-K) MDA-231 and Hs578T cells, as seen by the reduced number of Annexin V-FITC stained cells in the MDA-231 and Hs578T sh-K (mK) cells compared to Kaiso-depleted MDA-231 and Hs578T cells transfected with an empty (E) vector. Together these findings suggest that silencing Kaiso enhances the apoptosis of TNBC cells.

Our observation that Kaiso depletion caused increased apoptosis in TNBC cells was intriguing but contradictory to recent findings in other cell types (MEF and HEK293) where Kaiso was implicated as a pro-apoptotic protein, and promoter of p53-mediated apoptosis. ${ }^{31}$ Since the TNBC cells utilized in this study (MDA-231, Hs578T, MDA-157) contain a mutant (mut)-p53 gene ${ }^{32-34}$ compared to MEF and HEK293 cells that express wt-p53, ${ }^{31}$ we postulated that Kaiso's role in apoptosis was contingent on the status of $p 53$ rather than cell type per se. To test this hypothesis, we performed Annexin V-FITC staining of MCF-7 cells transiently overexpressing Kaiso. The MCF-7 BCa cell line was selected for these studies as it expresses low levels of Kaiso and wt-p53. As seen in Supplementary Figure 2B, transient overexpression of Kaiso in MCF-7 cells enhanced the apoptosis/death of these cells, as evidenced by more Annexin V-FITC stained cells in the Kaiso-overexpressing (mKaiso) MCF-7 cells compared to their parental (empty) counterparts, consistent with the findings of Koh et $a l^{31}$

Pro-apoptotic proteins are up-regulated in Kaisodepleted TNBC cells. As Kaiso expression promotes survival in TNBC cells expressing mut-p53 (Figure 3c), we hypothesized that the pro-survival role of Kaiso in TNBC cells is due to its interaction with mut-p53. To test this hypothesis, we performed co-immunoprecipitation experiments and found that Kaiso associated with mut-p53 in MDA-231 and Hs578T cells, although a stronger interaction was observed between Kaiso and mut-p53 in MDA-231 compared to Hs578T cells (Figure 4a). Wt-p53-expressing MCF-7 cells were also examined as a positive control to confirm Kaiso's interaction with wt-p53 (Supplementary Figure 3A) as previously reported. ${ }^{31}$ 
a
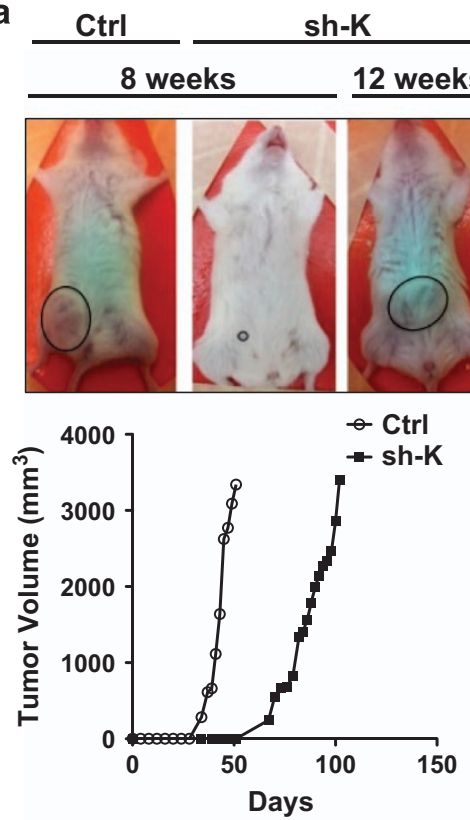

sh-K 12 weeks

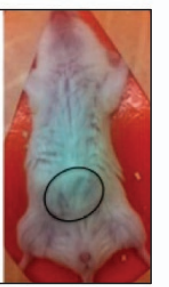

Ctrl

sh-K b
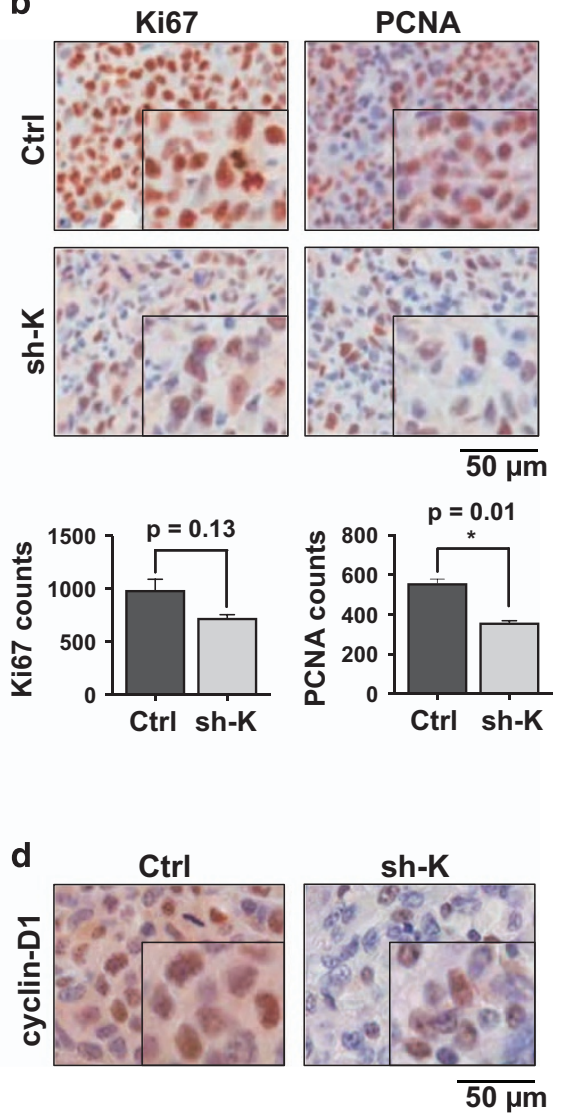

Figure 2 Kaiso-depleted MDA-231 cells exhibit delayed tumor onset in mouse xenografts. (a) Kaiso-depleted MDA-231 xenografts (sh-K) are delayed $\sim 3$ weeks in tumor onset and development compared to control (Ctrl) MDA-231 xenografted tumors as seen by time-course analysis of the tumor volume of Ctrl and sh-K MDA-231 xenografted cells. (b) IHC-stained images of MDA-231 xenograft tissues with Ki-67 and PCNA antibodies show a marked decrease in proliferating cells in MDA-231 Kaiso-depleted tumor tissues as indicated by the reduced expression of the proliferation markers Ki-67 and PCNA. (c and d) IHC-stained images of MDA-231 xenograft tissues with c-Myc and Cyclin D1 antibodies show that Kaiso-depletion results in reduced numbers of c-Myc and cyclin-D1 stained cells and reduced staining intensity. Representative images shown from 3 or more independent experiments

To gain more mechanistic insight into Kaiso's role in TNBC cell survival and apoptosis, we assessed the effect of Kaiso depletion on the expression of the pro-apoptotic proteins PUMA and Bax. As Kaiso augments the expression of PUMA and Bax in wt-p53-expressing cells ${ }^{31}$ and (Supplementary Figure 3B), we postulated that in mut-p53-expressing TNBC cells, Kaiso would inhibit PUMA and Bax expression. Indeed, low levels of PUMA were detected in control MDA-231 and Hs578T cells that express high levels of Kaiso and mut-p53 (Figure 4b). PUMA was also detected at low levels in the high Kaiso and mut-p53 MDA-157 cells (Supplementary Figure 4). Similarly, low Bax levels were detected in control MDA-231 and MDA-157 cells (Figure $4 \mathrm{~b}$ and Supplementary Figure 4) but not control Hs578T cells (Figure 4b). Remarkably, Kaiso depletion resulted in a striking upregulation of PUMA in all three cell lines (MDA-231, Hs578T and MDA-157 cells; Figure $4 \mathrm{~b}$ and Supplementary Figure 4 ). While Bax expression was increased $\sim 2$-fold in MDA-231 and MDA-157 cells, there was only a slight increase in Bax expression in Hs578T cells (Figure $4 \mathrm{~b}$ and Supplementary Figure 4), which may be due to the fact that Bax was expressed at higher levels in parental Hs578T cells compared to parental MDA-231 and MDA-157 cells (Figure $4 \mathrm{~b}$ and Supplementary Figure 4). This suggests that Kaiso may not exhibit a repressive function on Bax expression in parental (Ctrl) Hs578T cells, probably due to the

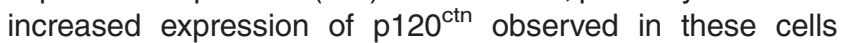
(Figure 4b), which co-localized with Kaiso in the nucleus of some but not all Ctrl Hs578T cells (Supplementary Figure 5). Notably, there was no change in mut-p53 levels upon Kaiso depletion. The specificity of Kaiso depletion effects on Bax and PUMA protein levels was confirmed by the expression of a sh-resistant Kaiso (mKaiso) cDNA in the MDA-231 sh-K cells; this resulted in reduced Bax and PUMA protein expression in the MDA-231-sh-K (mKaiso) cells (Figure 4c).

Additional analyses using qRT-PCR revealed significantly increased PUMA transcripts but no significant changes in Bax transcript levels in Kaiso-depleted MDA-231 and Hs578T cells compared to controls (Figure 4d). Consistent with this observation, chromatin immunoprecipitation (ChIP)-PCR experiments showed an enrichment of Kaiso at a minimal PUMA promoter region rich in Kaiso binding sequences (KBS) but not at a minimal Bax promoter region containing a core KBS (see schematic, Supplementary Figure 6A) in MDA-231 and Hs578T cells (Figure 4e, and data not shown). Similar 
a
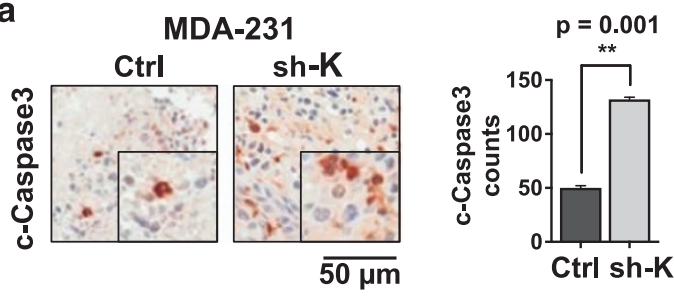

b

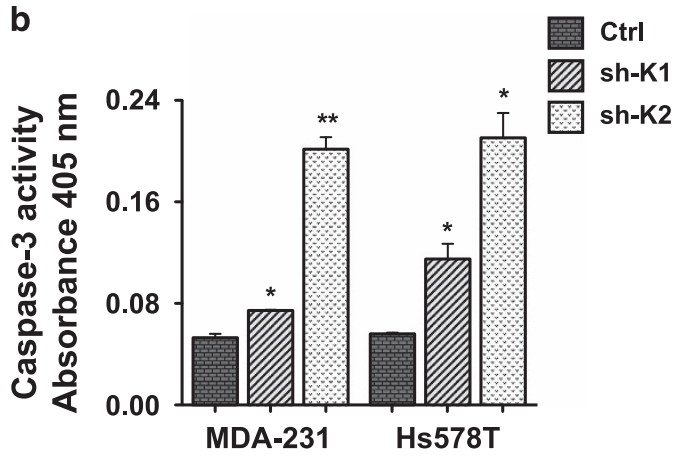

C
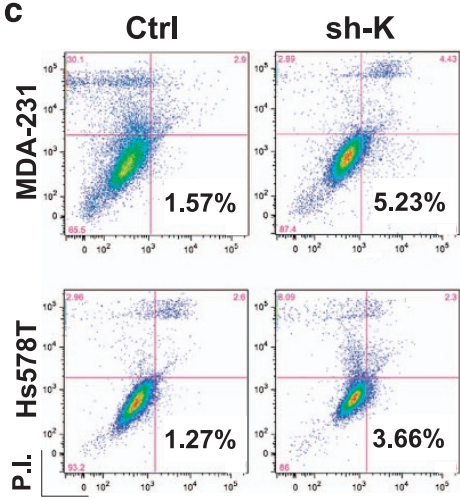

ANNEXIN V
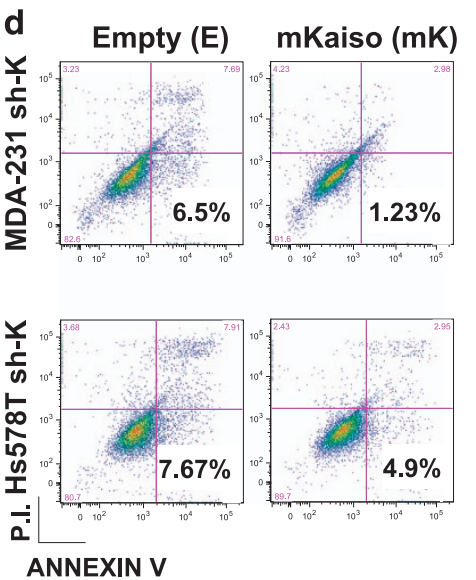
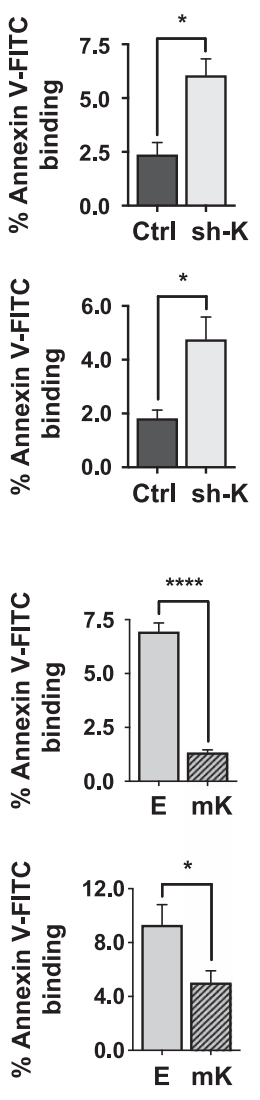

Figure 3 Kaiso depletion results in increased apoptosis of TNBC cells. (a) IHC-stained images of control (Ctrl) and Kaiso-depleted (sh-K) MDA-231 tumor tissues with cleaved (c)-Caspase 3 antibody show that Kaiso-depletion results in increased c-Caspase 3 expression in MDA-231 tumor tissues. (b) Caspase 3 assay conducted on Ctrl, sh-K1 and sh-K2 MDA-231 and Hs578T cells show that Kaiso depletion resulted in increased Caspase 3 activity in MDA-231 and Hs578T sh-K1 and sh-K2 cells compared to their control counterparts. (c) Kaiso-depleted TNBC cells (sh-K MDA-231 \& Hs578T) expressing mut-p53 exhibit increased apoptosis as revealed by Annexin V-FITC staining. (d) Expression of a sh-resistant Kaiso cDNA in Kaiso-depleted MDA-231 and Hs578T cells mitigates the apoptosis induced by Kaiso depletion as assessed by Annexin V-FITC staining. Data shown are representative of three independent experiments. ${ }^{*} P<0.05,{ }^{* *} P<0.01,{ }^{* \star * *} P<0.0001$

results were also obtained with chromatin isolated from MDA-157 cells (Supplementary Figure 6B). Interestingly, an enrichment of mut-p53 was also observed at the minimal PUMA promoter region rich in KBS but not at the minimal Bax promoter region containing a core KBS (Figure 4e, and data not shown). Nonetheless, Kaiso's interaction with the PUMA promoter was independent of p53 as evidenced by its association with the PUMA promoter in MDA-157 cells, which lack detectable p53 protein expression (Supplementary Figure 4). Collectively, these data imply that Kaiso may directly or indirectly inhibit Bax and PUMA expression in TNBC cells that lack wt-p53.

High Kaiso and low PUMA expression does not correlate with poor survival in $\mathrm{BCa}$ patients. Considering the consistent effect of Kaiso depletion on PUMA expression in all TNBC cell lines (MDA-231, Hs578T and MDA-157) studied, we explored whether the inverse correlation of Kaiso and PUMA expression could account for Kaiso's role in the survival of breast tumors. We thus utilized The Cancer Genome Atlas (TCGA) and the Gene Expression Omnibus (GEO) BCa data sets and examined the effect of high Kaiso and low PUMA expression on the overall survival of either TNBC patients specifically (data not shown), or all $\mathrm{BCa}$ cases. Kaplan-Meier survival curves revealed that patients bearing tumors with high Kaiso and low PUMA expression exhibited a decreased but non-significant overall survival trend compared to patients with tumors that had a low Kaiso and high PUMA expression (log-rank test, $P$-value $=0.16$; Figure 4f). This suggests that while Kaiso's effect on PUMA expression does have some effect on BCa survival, the clinical relevance does not appear to be statistically significant. Thus, Kaiso may cooperate with other protein(s) to influence BCa survival.

Kaiso depletion enhances the sensitivity of TNBC cells to Cisplatin. Most metastatic BCas such as TNBCs are resistant to chemotherapeutic agents, ${ }^{3}$ a phenomenon which may be due to reduced apoptosis and increased DNA repair. ${ }^{35}$ As Kaiso depletion stimulated the apoptosis of TNBC cells, we investigated whether silencing Kaiso would sensitize these cells to chemotherapeutic drugs. Control and Kaiso-depleted TNBC cells were treated with the chemotherapy drug Cisplatin and then subjected to immunoblot (IB) 


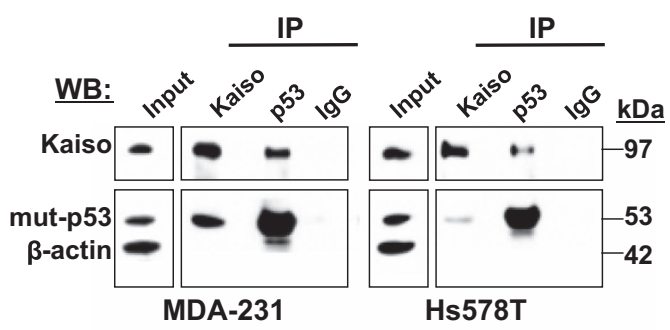

c

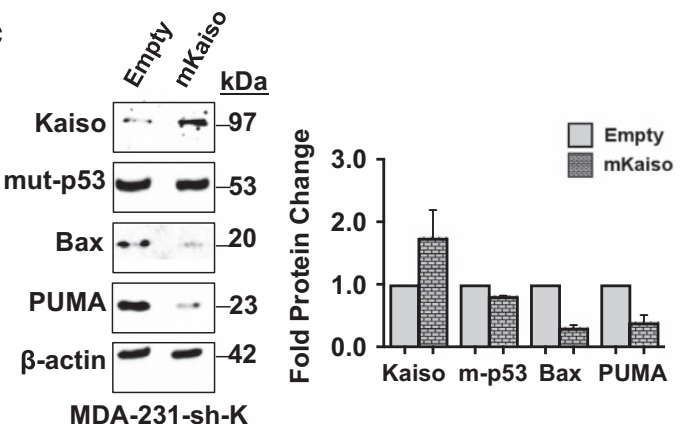

d
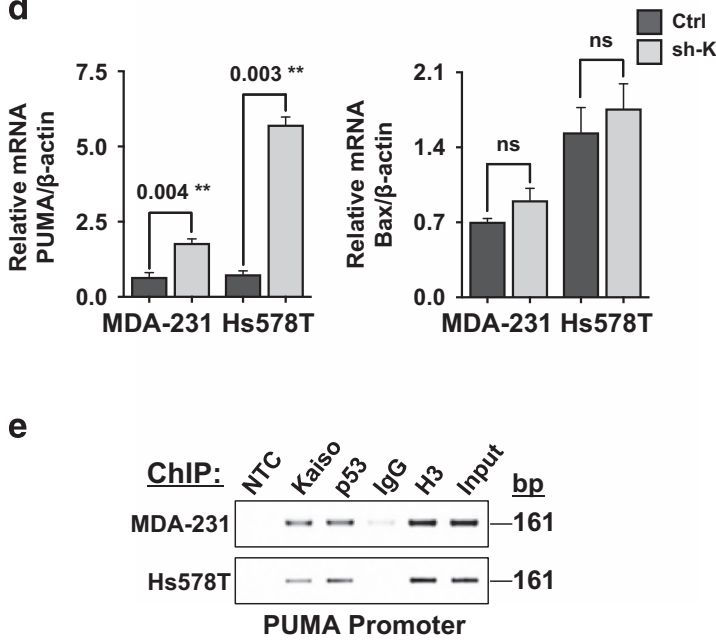

b
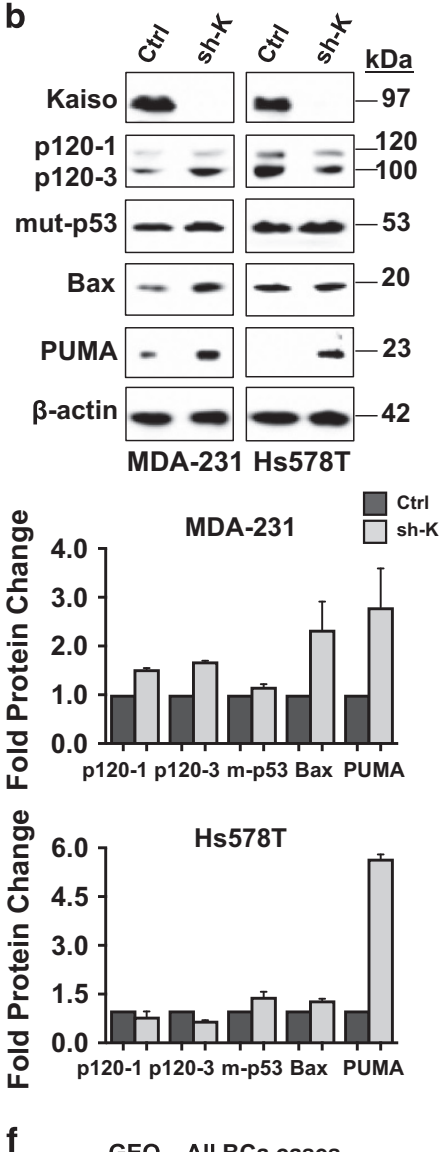

f

GEO - All BCa cases

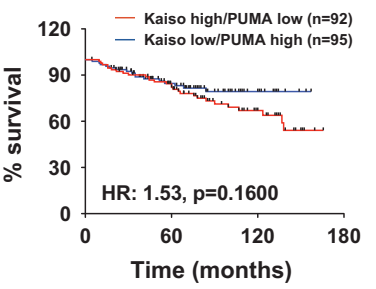

Figure 4 Kaiso depletion increases expression of pro-apoptotic proteins in TNBC cells lacking wt p53. (a) Kaiso co-precipitates with mutant p53 in MDA-231 and Hs578T cell lysates. Parental MDA-231 and Hs578T cells were subjected to immunoprecipitation with anti-Kaiso, anti-p53 and anti-lgG antibodies, and immunoblotted with the indicated antibodies. Kaiso-depleted MDA-231 and Hs578T cells express increased amounts of Bax and PUMA protein compared to control cells (b), that is decreased upon expression of a sh-resistant Kaiso cDNA in the MDA-231 and Hs578T sh-K cells (c). Graphical representation of the quantitated protein values is shown. (d) Kaiso-depleted MDA-231 and Hs578T cells (that express mut-p53) exhibit a statistically significant increase in PUMA mRNA levels as measured by qRT-PCR. Although Bax mRNA levels were also slightly increased, it was not significant. (e) ChIP-PCR analysis of MDA-231 and Hs578T chromatin revealed that Kaiso and mut-p53 associate endogenously with the PUMA promoter in TNBC cells. (f) Transcript profiles of patients from the GEO (GSE20685, GSE21653, GSE16446, GSE19615 and GSE9195) BCa data sets were pooled and segregated into Kaiso high/PUMA low, and Kaiso low/PUMA high groups. Kaplan-Meier survival curves revealed a correlation between high Kaiso and low PUMA expression with poor overall survival in all BCa cases. However, it was not statistically significant. Data representative of three independent experiments. ${ }^{* *} P<0.01$ and NS, not significant. For Kaplan-Meier survival curves, log-rank test was performed to determine statistical significance. $P<0.05$ is considered statistically significant

analysis for the expression of the apoptotic marker, cleaved-PARP. Intriguingly, loss of Kaiso enhanced the sensitivity of MDA-231, Hs578T and MDA-157 cells to Cisplatin as evidenced by the increased expression of cleaved-PARP in the treated Kaiso-depleted cells compared to the control-treated cells (Figures $5 a$ and $b$ ).

High Kaiso and BRCA1 expression correlates with poor survival in $\mathrm{BCa}$ patients. As BRCA1 expression has been linked to the resistance of TNBC cells to Cisplatin, ${ }^{10,36}$ we examined the effect of Kaiso depletion on BRCA1 expression. We observed that Kaiso depletion led to downregulation of BRCA1 in MDA-231 and Hs578T cells at both the transcript and protein level (Figures $6 a$ and b). This was partially rescued ( 1.6-fold increase) by the expression of a sh-resistant Kaiso cDNA (mKaiso) in the Kaiso-depleted MDA-231 and Hs578T cells (Figure 6c). More importantly, we found an enrichment of Kaiso at a minimal BRCA1 promoter region containing several core KBS (Figure 6d), which suggests that BRCA1 may be a Kaiso target gene. 
In light of these findings, we utilized the TCGA and GEO $\mathrm{BCa}$ data sets and correlated the expression levels of Kaiso and BRCA1 with BCa survival. Kaplan-Meier survival curves revealed that TNBC patients bearing tumors with high Kaiso and BRCA1 expression, exhibit a significantly worse overall

a
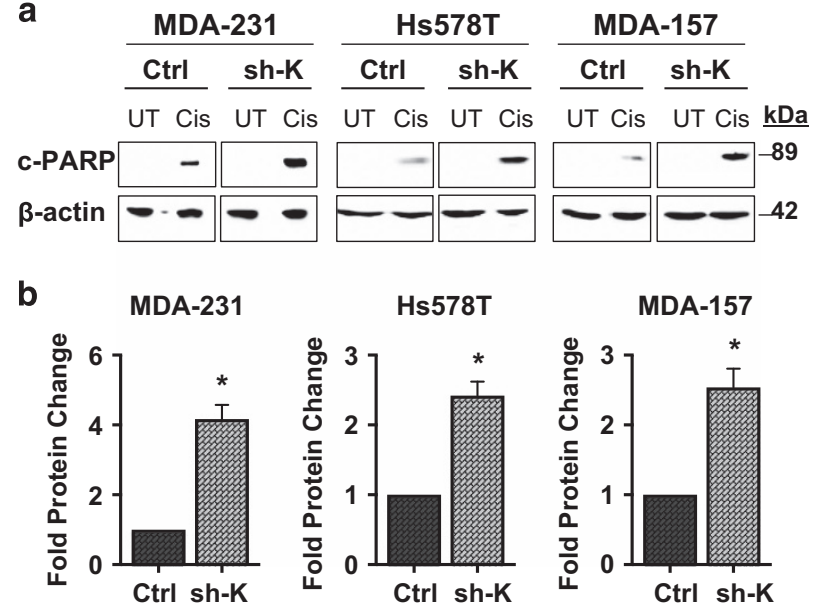

Figure 5 Kaiso depletion enhances the sensitivity of TNBC cells to Cisplatin. (a) Kaiso-depletion sensitizes TNBC cells to Cisplatin treatment, as demonstrated by the increased cleaved-PARP expression observed in Kaiso-depleted MDA-231, Hs578T and MDA-157 cells treated with Cisplatin (Cis) for $48 \mathrm{~h}$ compared to similarly treated control cells. UT, untreated cells. (b) Graphical representation of the fold change in protein expression is shown. All experiments were conducted independently at least three times. Representative images shown. ${ }^{*} P<0.05$ survival compared to TNBC patients with low Kaiso and low BRCA1 expression (log-rank test, $P=0.017$; Figure 7a). A similar trend was also observed in all BCa cases with high Kaiso and high BRCA1 expression (log-rank test, $P=0.0003$ ) compared to cases with low Kaiso and low BRCA1 expression, and high or low BRCA1 expression alone (log-rank test, $P=0.13$ ), Figures $7 \mathrm{~b}$ and $\mathrm{c}$. This finding suggests that Kaiso and BRCA1 function together to promote the survival of $\mathrm{BCa}$ cells.

\section{Discussion}

TNBCs remain a clinical challenge due to their highly aggressive nature, lack of specific targeted therapies and resistance to routine chemotherapeutic regimens including anthracyclines and taxanes. ${ }^{37}$ Consequently, there is an urgent need to understand the molecular mechanisms underlying TNBC growth, aggressiveness and chemo-resistance. Herein, we report that depletion of the transcription factor Kaiso attenuates the proliferation of, and increases apoptosis in, the TNBC cell lines MDA-231 and Hs578T. These findings suggest that in addition to Kaiso's potential role in TNBC metastasis, ${ }^{26}$ Kaiso may also be a key regulator of triple negative tumor cell growth and survival.

In the past decade, several independent studies have implicated Kaiso in various cancers; while some studies suggest a pro-oncogenic role for Kaiso, ${ }^{19,20,26,28,38-42}$ others associate Kaiso with a tumor suppressive role. ${ }^{23,25,31,43}$ Together, these diverse studies highlight context-dependent roles for Kaiso in human cancer, which might be due to the fact
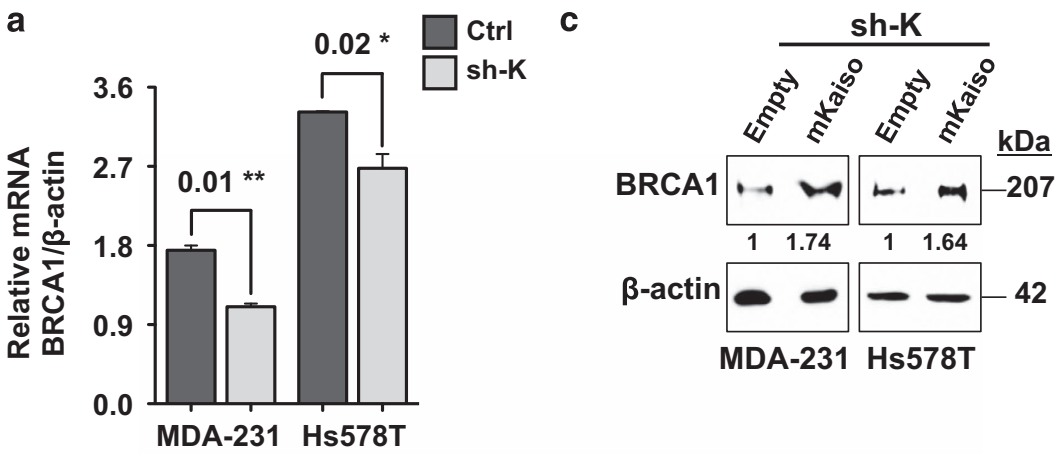

b

d
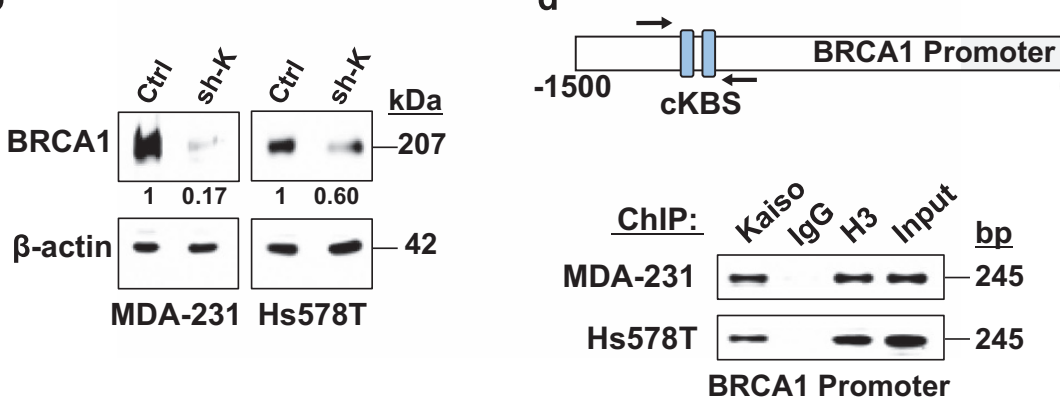

Figure 6 Kaiso depletion attenuates BRCA1 expression in sporadic TNBC cells. (a) BRCA1 mRNA expression was significantly reduced in Kaiso-depleted MDA-231 and Hs578T cells compared to controls as measured by qRT-PCR. (b) WB analysis with a BRCA1-specific antibody shows decreased BRCA1 protein expression in Kaiso-depleted MDA-231 and Hs578T cells, which is partially rescued by expression of a sh-resistant Kaiso cDNA in the MDA-231 and Hs578T sh-K cells (c). (d) Schematic illustration of the minimal BRCA1 promoter region showing the location of a core KBS (CKBS) that was amplified by ChIP-PCR. Kaiso was enriched at the BRCA1 promoter region indicated in MDA-231 and Hs578T cells. Data representative of three independent experiments. ${ }^{*} P<0.05,{ }^{\star *} P<0.01$ 
TCGA - TNBC cases

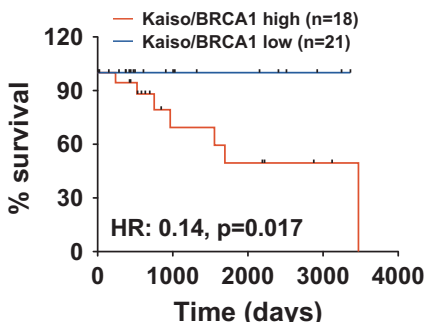

b

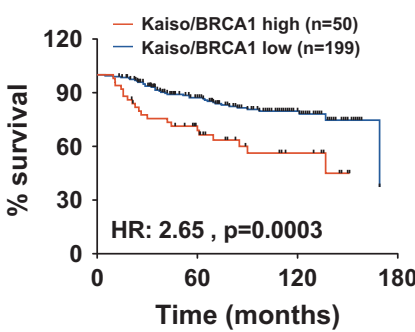

C

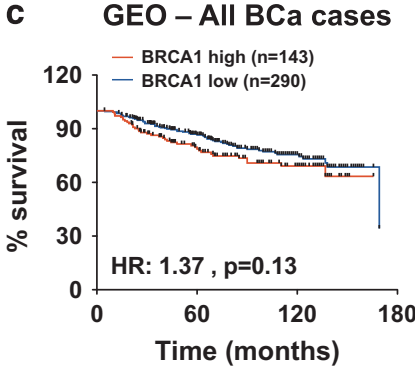

Figure 7 High Kaiso and BRCA1 expression correlates with poor prognosis in BCa patients. Transcript profiles of patients from the TCGA and GEO (GSE20685, GSE21653, GSE16446, GSE19615 and GSE9195) BCa data sets were pooled and segregated into Kaiso/BRCA1 high or Kaiso/BRCA1 low, and BRCA1 high or BRCA1 low groups. KaplanMeier survival curves revealed that high Kaiso and BRCA1 expression significantly correlates with poor overall survival in TNBC patients specifically (a) or all BCa cases (b) whereas increased BRCA1 expression did not correlate with poor overall survival in $\mathrm{BCa}$ patients (c). Log-rank test was performed to determine statistical significance. $P<0.05$ is considered statistically significant

that Kaiso acts as both a transcriptional repressor and an activator. $^{24,29,30,44,45}$ In addition, as Kaiso also possesses dual-specificity DNA-binding properties, ${ }^{29,46,47}$ there may be a large repertoire of tumorigenic target genes that may be differentially regulated by Kaiso. To date, only a few bona fide Kaiso target genes-c-Myc, Wnt 11, Cyclin D1, Siamois, Matrilysin and Rapysn have been characterized. 24,25,29,45,48 Two of these genes (c-Myc and Cyclin D1) are wellestablished pro-proliferation oncogenes ${ }^{49,50}$ that were found to be repressed by Kaiso in Xenopus laevis embryos and colon cancer cells. ${ }^{24,29}$ Therefore, it was surprising to find that loss of Kaiso in TNBC cells led to their decreased, rather than increased, expression (Figure 1e). Our findings thus indicate context-dependent roles for Kaiso in the regulation of $c-M y c$ and Cyclin D1 expression, an idea that is supported by a recent study which demonstrates differential regulation of Cyclin $D 1$ by Kaiso. ${ }^{27}$

Kaiso's role in specific cancers may also be dictated or modulated by its interaction with other transcriptional cofactors or proteins that may be uniquely expressed in these cancers. For example, Kaiso was shown to interact with nuclear $\mathrm{p} 120^{\mathrm{ctn}}$ in mouse invasive lobular BCa cells, which inhibited Kaiso's repression of Wnt11, and fostered anoikis resistance in these cells. ${ }^{23}$ In another study, Kaiso was shown to interact with wt-p53, and promote apoptosis through increased p53-mediated expression of the pro-apoptotic Bax and PUMA genes. ${ }^{31}$ Our findings in this study also support distinct roles of Kaiso that may be based on its interaction with p53, as we found that Kaiso differentially regulates apoptosis in BCa cells that express different forms of p53 (Figure 3c, Supplementary Figures $2 A$ and B). Specifically, Kaiso exhibits an anti-apoptotic role in TNBC (MDA-231, Hs578T and MDA-157) cells that express mut-p53 as its depletion promotes apoptosis in these cells (Figure $3 \mathrm{c}$ and Supplementary Figure 2A). As mut-p53 expression is implicated in the survival of MDA-231 and Hs578T cells, ${ }^{51,52}$ it was interesting to note that loss of Kaiso attenuated the survival of these cells, despite having no significant effects on mut-p53 expression in these cells.

Conversely, in non-TNBC cells that express wt-p53, Kaiso exhibits a pro-apoptotic role (Supplementary Figure 2B), which is consistent with reports in other cell types that demonstrated a pro-apoptotic role for Kaiso in a wt-p53-dependent a

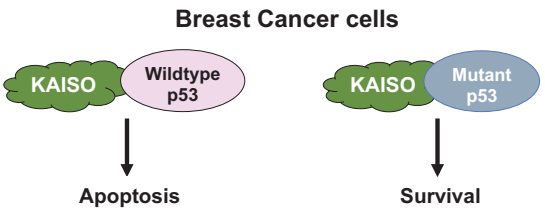

b

b Triple Negative Breast Cancer cells

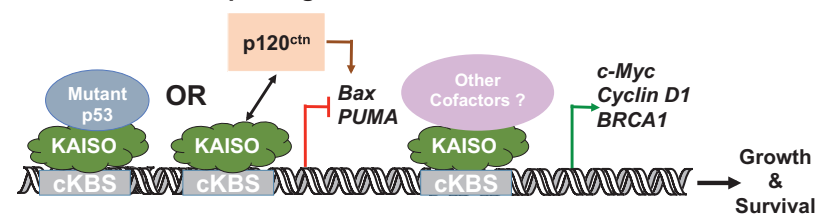

Figure 8 Schematic diagram of proposed model for Kaiso's role in TNBC. (a) Kaiso interacts with both wt p53 and mutant p53 in BCa cells and this differential interaction may modulate Kaiso's function in apoptosis. (b) In TNBC cells lacking wt p53 (but expressing mutant p53), Kaiso might directly or indirectly inhibit the activation of the pro-apoptotic genes Bax and PUMA, which leads to tumor survival. However, Kaiso's inhibitory effect on Bax protein expression may be attenuated by Kaiso interaction with other proteins like $\mathrm{p} 120^{\mathrm{ctn}}$. Kaiso may also activate c-Myc, Cyclin D1 and BRCA1 expression independently or in collaboration with other cofactors in TNBC cells, which would also promote TNBC cell growth and survival

manner. ${ }^{31}$ Based on these findings, we surmise that the distinct roles of Kaiso in apoptosis may be due to its ability to interact with both wt-p53 and mut-p53 as shown in Figure $4 a$ and Supplementary Figure $3 A$ (see model indicated in Figure 8a). Indeed, several recent studies have reported differential activities of transcription factors that interact with both wt-p53 and mut-p53, reviewed in Kim et al. ${ }^{53}$ As Kaiso behaved in an anti-apoptotic manner in TNBC cells lacking wt-p53, we postulate that Kaiso may only function in a pro-apoptotic manner in BCa cells expressing wt-p53.

An unexpected finding of this study was that Kaiso depletion reduced $\mathrm{BRCA} 1$ expression at both the transcript and protein levels in TNBC cells, suggesting that BRCA1 may be a Kaiso target gene. Indeed, we observed that Kaiso associates with the BRCA1 promoter in both MDA-231 and Hs578T cells (Figure 6d) but more importantly, we also found that high Kaiso and BRCA1 expression correlates with poor overall survival in TNBC patients, as well as all BCa cases in general (Figures 7a and b). Collectively, our findings suggest that Kaiso may 
augment the survival and aggressiveness of TNBC cells by promoting BRCA1 expression (see model, Figure 8b). Hence, our demonstration that Kaiso-depletion enhanced the sensitivity of TNBC cells to the chemotherapy drug Cisplatin raise the exciting possibility that Kaiso may be a target for TN tumors with BRCA1 expression.

Together, this study reveals an essential role for Kaiso in the growth and survival of TNBC cells and suggests that Kaiso could be targeted for the treatment of a subset of triple negative tumors especially those expressing BRCA1. Future experiments (e.g. ChIP-sequencing and RNA-sequencing of control and Kaiso-depleted TNBC cells) are needed to fully delineate and understand the molecular mechanisms and signaling pathways that Kaiso participates in to contribute to the pathogenesis and survival of triple negative tumors.

\section{Materials and Methods}

Cell culture. The human triple negative breast tumor cell lines MDA-MB-231 (hereafter MDA-231) and Hs578T, and their stable Kaiso-depleted (sh-K1 and sh-K2) derivatives were cultured as previously described. ${ }^{26}$ The non-TNBC cell line MCF-7 was purchased from ATCC (Manassas, VA, USA), while the triple negative breast tumor cell line MDA-MB-157 (hereafter MDA-157) and the non-TNBC cell line ZR75.1 were a kind gift from Dr. John Hassell (McMaster University, Hamilton, Canada). These cells were cultured as previously described. ${ }^{54}$ All cells were grown in $5 \% \mathrm{CO}_{2}$ at $37^{\circ} \mathrm{C}$.

Generation of stable Kaiso-depleted MDA-157 cell lines. Depletion of Kaiso in MDA-157 cells was achieved by stably transfecting cells with a pRetroSuper (pRS) vector containing shRNAs that targeted the Kaiso mRNA sequences; 5'-AAAAGATCATTGTTACCGATT-3' and 5'-TTTTAACATTCATTCTTG GGAGAAG-3' termed sh-K1 and sh-K2 as previously described. ${ }^{26}$ Stable control (transfected with a pRS-Kaiso scrambled shRNA) ${ }^{26}$ and Kaiso-depleted MDA-157 cells were maintained in media containing $1.0 \mu \mathrm{g} / \mathrm{ml}$ of Puromycin (Invitrogen, Carlsbad, CA, USA). Only the most efficient Kaiso-depleted cells were selected for further analysis (Supplementary Figure 1b).

Cell proliferation assay. Equal numbers $\left(1 \times 10^{4}\right)$ of control and Kaisodepleted (sh-K1 and sh-K2) MDA-231 and Hs578T cells were grown in 24-well plates for 3 days. Direct cell counts were obtained each day using the BioRAD TC10 automated cell counter and averaged using Microsoft Excel. Graphical representation of counts was achieved using GraphPad Prism software (La Jolla, CA, USA).

MTT assay. Equal numbers $\left(1 \times 10^{4}\right)$ of control and Kaiso-depleted (sh-K1 and sh-K2) MDA-231 and Hs578T cells were grown in duplicate in 96-well plates for $22 \mathrm{~h}$. Cells were then immediately treated with MTT (3-(4,5-dimethylthiazol-2-yl)-2, 5-diphenyl-tetrazolium bromide; Sigma Aldrich, USA) for $2 \mathrm{~h}$. The precipitated formazan crystals were subsequently dissolved with $100 \mu \mathrm{l}$ of dimethyl sulfoxide and the optical density of the resulting reaction solution measured at $570 \mathrm{~nm}$ using the SpectraMax Plus 384 Microplate reader (Molecular Devices, Sunnyvale, CA, USA).

Colony formation and soft agar assay. $5 \times 10^{2}$ control and Kaisodepleted MDA-231 and Hs578T cells were cultured in $60 \mathrm{~mm}$ dishes in duplicate and allowed to grow and form colonies for 10-14 days. For the soft agar assays, $5 \times 10^{4}$ control and Kaiso-depleted MDA-231 and Hs578T cells were cultured in $0.3 \%$ Agarose in $60 \mathrm{~mm}$ dishes, and allowed to grow and form colonies for 10 days. After the incubation period, colonies were stained with 0.5 and $0.05 \%$ Gentian Violet diluted in methanol for the colony formation and soft agar assays respectively. Images of colonies from the colony formation assay were obtained by using a Canon digital camera and then colonies were counted manually. For the soft agar assay, 10x images of colonies were obtained using the Zeiss Axiovert 200 microscope (Carl Zeiss Canada Ltd., ON, Canada), and then counted using the ImageJ software. Graphical representation of counts (colony numbers) was achieved using GraphPad Prism software.
Xenograft studies. All mice studies were approved by the Animal Research Ethics Board, McMaster University (AUP\# 14-05-14) and performed in accordance with the guidelines of the Animal Research Ethics Board as previously described. ${ }^{26}$ In brief, equal numbers $\left(4.5 \times 10^{6}\right)$ of control and Kaiso-depleted MDA-231 cells were injected subcutaneously into the mammary fat pad of $\sim 5$-8-week-old female NOD SCID gamma mice ( $n=5$ each) and allowed to form tumors. Tumor growth was monitored using vernier calipers and tumor volume measurements calculated as previously described. ${ }^{26}$ Mice were euthanized at endpoint (tumor size $3300 \mathrm{~mm}^{3}$ ) as previously described ${ }^{26}$ and tumor tissues harvested for histological examination and IHC analyses.

Immunohistochemistry. $5 \mu \mathrm{M}$ sections of harvested MDA-231 xenografted tumor tissues were stained with mouse monoclonal antibody against Ki-67 (BD Biosciences; 1:50), rabbit monoclonal antibody against PCNA (Cell Signaling Technology (CST)-D3H8P; 1:30 000), rabbit monoclonal antibody against C-Myc (Abcam; 1:100), rabbit monoclonal antibody against Cyclin D1 (CST-2978; 1:100) or rabbit monoclonal antibody against cleaved Caspase 3 (CST-9661; 1:50) overnight at $4{ }^{\circ} \mathrm{C}$ as previously described. ${ }^{26}$ Images were captured using the Aperio Slide scanner (Leica Biosystems, ON, Canada). Ki-67, PCNA and cleaved Caspase 3 counts were obtained from 5 different fields that represented staining observed in whole-tissue sections. The stained cells in these fields were counted blindly and independently by 2 students. Bar graphs representing counts were generated using GraphPad Prism software. Statistically analyses were also conducted using GraphPad Prism statistical software.

Transient transfection assay and rescue experiments. MCF-7 and ZR75.1 parental cells were transfected with either a pCDNA3-empty vector (empty), or a pCDNA3 vector containing the sequence that encodes a sh-resistant mKaiso cDNA using the Turbofect transfection reagent (Thermo Scientific, Waltham, MA, USA) according to the manufacturer's instructions. 48 or $72 \mathrm{~h}$ post transfection, cells were either subjected to IB analysis, or treated with Geneticin (Invitrogen) at $250 \mu \mathrm{g} / \mathrm{ml}$ for MCF-7 cells and $750 \mu \mathrm{g} / \mathrm{ml}$ for ZR75.1 cells to select for efficient Kaiso overexpression. Three to four weeks post transfection, whole-cell lysates were obtained from the pCDNA3-empty and mKaiso transfected cells and subjected to IB analysis of interested proteins.

For rescue of Kaiso overexpression, pCDNA3 vector expressing the mKaiso cDNA coding sequence that is not targeted by the Kaiso-specific shRNA was transfected into MDA-231 and Hs578T sh-K2 (or sh-K) cells using the Turbofect transfection reagent (Thermo Scientific) as per the manufacturer's protocol. 24 or $48 \mathrm{~h}$ post transfection, cells were treated with Puromycin (Invitrogen) at $0.8 \mu \mathrm{g} / \mathrm{ml}$ and Geneticin (Invitrogen) at $1000 \mu \mathrm{g} / \mathrm{ml}$ for MDA-231 sh-K cells and Puromycin (Invitrogen) at $1.5 \mu \mathrm{g} / \mathrm{ml}$ and Geneticin (Invitrogen) at $1000 \mu \mathrm{g} / \mathrm{ml}$ for Hs578T sh-K cells to select for efficient Kaiso overexpression. Three to four weeks post transfection, MDA-231 and Hs578T sh-K (empty and mKaiso) cells were subjected to Annexin V-FITC staining. Whole-cell lysates were also obtained from MDA-231 and Hs578T sh-K (empty and mKaiso) cells and subjected to IB analysis of interested proteins.

Caspase 3 assay. The Caspase 3 assay (colorimetric) kit was purchased from Abcam (Boston, MA, USA), and the assay performed according to the manufacturer's instructions. In brief, $1 \times 10^{6}$ control and Kaiso-depleted (sh-K1 and sh-K2) MDA-231 and Hs578T cells were re-suspended in $50 \mu \mathrm{l}$ of chilled cell lysis buffer, incubated on ice for $10 \mathrm{~min}$ and pelleted by centrifugation at 13000 r.p.m. for $1 \mathrm{~min}$. The resulting supernatant (cytosolic extract) was then transferred to a new tube, quantified and then $\sim 200 \mu \mathrm{g}$ of protein per $50 \mu \mathrm{l}$ cell lysis buffer transferred into 96-well plates in duplicate per cell condition. $50 \mu \mathrm{l}$ cell lysis buffer without protein samples was also aliquoted into 96-well plates to provide background readings. $50 \mu \mathrm{l}$ reaction buffer $(2 x)$ containing $10 \mathrm{mM}$ DTT was added to each well containing experimental samples (in duplicate) and cell lysis buffer (without samples) followed by $5 \mu \mathrm{l}$ of $4 \mathrm{mM}$ DEVD-p-NA substrate $(200 \mu \mathrm{M}$ final concentration). The resultant mixture was incubated at $37^{\circ} \mathrm{C}$ for $2 \mathrm{~h}$, and then the optical density of the solution was measured at $405 \mathrm{~nm}$ using the SpectraMax Plus 384 Microplate reader (Molecular Devices).

ANNEXIN V-FITC staining assay. The FITC-conjugated Annexin V apoptosis detection kit was purchased from Abcam, and staining performed according to the manufacturer's instructions. In brief, equal numbers $\left(1 \times 10^{5}\right)$ of $\mathrm{Ctrl}$ and sh-K MDA-231, Hs578T and MDA-157 cells, MDA-231 and Hs578T sh-K (empty and mKaiso) cells, as well as MCF-7 (empty and mKaiso) cells were 
re-suspended in $1 \times$ binding buffer (Abcam) after being washed with $1 \times$ PBS and trypsinized with $1 \times$ Trypsin (Invitrogen). These cells were subsequently stained with Annexin V-FITC and propidium iodide (PI) and incubated for $10 \mathrm{~min}$ prior to analysis by Flow cytometry. Data were acquired using the LSRFortessa flow cytometer (BD Biosciences, Mississauga, Canada) and analyzed with FlowJo version 9 software.

Quantitative reverse transcription-PCR (qRT-PCR). qRT-PCR experiments were conducted as previously described ${ }^{26}$ using the following primers: Bax forward: $5^{\prime}$-GCCCTTTTGCTTCAGGGTTT- $3^{\prime}$ and reverse: $5^{\prime}$-GCAATCATCCTCT GCAGCTC- $3^{\prime}$ at $60^{\circ} \mathrm{C}$, PUMA forward: $5^{\prime}$-AGCAGGGCAGGAAGTAACAA- $3^{\prime}$ and reverse: $5^{\prime}$-CCCTGGGGCCACAAATCT- $3{ }^{\prime}$ at $55^{\circ} \mathrm{C}$, BRCA1 forward: $5^{\prime}$-CTCGCTG AGACTTCCTGGAC-3' and reverse: 5'-TCAACTCCAGACAGATGGGAC-3' at $62{ }^{\circ} \mathrm{C}$. The SensiFAST cDNA synthesis kit and the SensiFAST SYBR Hi-ROX kit (FroggaBio Scientific Solutions, Toronto, ON, Canada) were used in place of the qScript cDNA SuperMix and Perfecta SYBR Green SuperMix ROX (Quanta BioSciences, Gaithersburg, MD, USA) as previously described. ${ }^{26}$

ChIP and ChIP-PCR. ChIP and ChIP-PCR were performed as previously described ${ }^{26}$ The following primers were used to amplify a minimal Bax, PUMA and BRCA1 promoter region, respectively, containing one or more KBS: Bax KBS forward: 5'-CTAATTCCTTCTGCGCTGGG-3', and reverse: $5^{\prime}$-GTCCAATCGCAGCT CTAATGC- $3^{\prime}$ at $64^{\circ} \mathrm{C}$; PUMA KBS forward: $5^{\prime}$-GATCGAGACCATCCTGGCTA-3' and reverse: $5^{\prime}$-CGATCTCAGCAAACTGCAAG- $3^{\prime}$ at $64^{\circ} \mathrm{C}$; and BRCA1 KBS forward: $5^{\prime}$-AGGGCTCTCTCATCCTGTCA-3' and reverse: $5^{\prime}$-TGTCCGCCATGT TAGATTCA-3' at $64^{\circ} \mathrm{C}$.

Immunoprecipitation. Whole-cell lysates were immunoprecipitated with antiKaiso 6F mouse monoclonal antibody, ${ }^{55}$ anti-p53 mouse monoclonal antibody (CST-2524, which recognizes both wt and mutant-p53), anti-p53 rabbit monoclonal antibody (Abcam-ab32049 that recognizes only mutant-p53) or normal rabbit lgG antibody (Santa Cruz Technology) for $2 \mathrm{~h}$ or overnight at $4{ }^{\circ} \mathrm{C}$. The immunoprecipitates were collected by incubation with $50 \mu \mathrm{l}$ Protein A agarose beads that were subsequently washed five times with lysis buffer before proceeding to SDS-PAGE and IB analysis.

Immunoblot and densitometry analysis. IB analysis was performed as previously described. ${ }^{26}$ Overnight incubations were performed at $4{ }^{\circ} \mathrm{C}$ using the following primary antibodies at their respective dilutions; Kaiso-specific rabbit polyclonal (gift from Dr. Reynolds; 1:5000), mouse monoclonal antibody against C-Myc (SantaCruz (9E10); 1:500), rabbit polyclonal antibody against Cyclin D1 (US Biological (144418); 1:5000), p120 $0^{\text {ctn }}-15 D 2$ specific mouse monoclonal (gift from Dr. Reynolds; 1:1000 ${ }^{56}$ ), Bax-specific rabbit monoclonal (1:500; CST-5023), PUMA-specific rabbit monoclonal (1:500; CST-12450), p53-specific rabbit polyclonal (1:2000; Abcam-ab32049), cleaved PARP-specific rabbit monoclonal (1:1000; CST-5625), BRCA1-specific rabbit polyclonal (1:2000; Abcamab131360) and mouse anti- $\beta$-actin monoclonal (1:50 000; Sigma Aldrich). IB images were obtained using the Bio-Rad ChemiDoc MP imaging system (Bio-Rad Laboratories, Mississauga, ON, Canada). The optical densities of Kaiso, c-Myc, Cyclin D1, p120-1, p120-3, p53, Bax, PUMA and $\beta$-actin signals were quantified and analyzed using the Image Lab software (Bio-Rad), while the relative ratio of Kaiso/ $\beta$-actin, $c$-Myc/ $\beta$-actin, Cyclin D1/ $\beta$-actin, $p 120-1 / \beta$-actin, $p 120-3 / \beta$-actin, p53/ $\beta$-actin, Bax $/ \beta$-actin and PUMA $/ \beta$-actin were calculated as indicated using Microsoft Excel. Graphical representation of each respective value was accomplished using GraphPad Prism software.

Gene expression analysis of GEO data sets. Gene expression analyses were conducted on five publicly available data sets obtained using Affymetrix HG-U133 plus 2.0 gene chip arrays (Affymetrix, Santa Clara, CA, USA). The transcript profiles of these data sets were deposited in the GEO database under accession numbers GSE20685, GSE21653, GSE16446, GSE19615 and GSE9195. ${ }^{57-62}$ All samples used for this study were normalized with frozen robust multi-array analysis ${ }^{63}$ and then the distance-weighted discrimination method ${ }^{64}$ was used to remove technical variation from the data sets that were to be combined. The combined data sets correlation coefficients for pair-wise comparisons of samples using Affymetrix house-keeping probe sets were computed, and only samples exhibiting a correlation higher than 0.95 with at least half of the data set were selected for further classification. This resulted in a cohort containing 894 tumor samples, which was subsequently used for generating Kaplan-Meier survival curves and performing log-rank analysis.
Gene expression analysis of TCGA data sets. TCGA Level 3 IlluminaHiSeq_RNASeqV2 expression (Illumina, Inc., San Diego, CA, USA) and associated clinical data were downloaded for all available patients from the Broad GDAC Firehose repository (https://gdac.broadinstitute.org/) on 16 September 2016 $(n=1212)$. In all further analyses this data set is referred to as 'TCGA dataset'. We selected tumor samples only $(n=1,094)$, and their RSEM-quantified gene expression values were log2-transformed and used for further analyses to represent gene expression. For identification of ER, PR and ERBB2 status and for overall survival information we used the downloaded clinical data. All data processing was performed using $R$ software. ${ }^{65}$

Survival analysis. Survival analysis and visualization of the Kaplan-Meier curves were performed using GraphPad Prism statistical software (GraphPad Software, Inc., La Jolla, CA, USA). For statistical tests $P$-value $<0.05$ indicated significance.

Statistical analyses. All statistical analyses were performed as previously described $^{26}$ using the GraphPad Prism software (GraphPad Software, Inc., La Jolla, CA, USA). $P<0.05$ values were considered statistically significant and data are presented as means \pm S.E.M.

\section{Conflict of Interest}

The authors declare no conflict of interest.

Acknowledgements. JMD was funded by the Canadian Breast Cancer Foundation (CBCF) and an NSERC Discovery Grant \# RGPIN-238700. BBA was funded by a Schlumberger Faculty for the Future Fellowship.

1. Foulkes WD, Smith IE, Reis-Filho JS. Triple-negative breast cancer. N Engl J Med 2010; 363: 1938-1948.

2. Brouckaert $\mathrm{O}$, Wildiers $\mathrm{H}$, Floris $\mathrm{G}$, Neven $\mathrm{P}$. Update on triple-negative breast cancer: prognosis and management strategies. Int $J$ Womens Health 2012; 4: 511-520.

3. O'Reilly EA, Gubbins L, Sharma S, Tully R, Guang MH, Weiner-Gorzel K et al. The fate of chemoresistance in triple negative breast cancer (TNBC). BBA Clin 2015; 3: 257-275.

4. Hill SJ, Clark AP, Silver DP, Livingston DM. BRCA1 pathway function in basal-like breast cancer cells. Mol Cell Biol 2014; 34: 3828-3842.

5. Hongthong K, Ratanaphan A. BRCA1-associated triple-negative breast cancer and potential treatment for ruthenium-based compounds. Curr Cancer Drug Targets 2016; 16: 606-617.

6. Varna M, Bousquet G, Plassa LF, Bertheau P, Janin A. TP53 status and response to treatment in breast cancers. J Biomed Biotechnol 2011; 2011: 284584

7. Ouchi $T$, Monteiro AN, August A, Aaronson SA, Hanafusa H. BRCA1 regulates p53-dependent gene expression. Proc Natl Acad Sci USA 1998; 95: 2302-2306.

8. Wong-Brown MW, Meldrum CJ, Carpenter JE, Clarke CL, Narod SA, Jakubowska A et al. Prevalence of BRCA1 and BRCA2 germline mutations in patients with triple-negative breast cancer. Breast Cancer Res Treat 2015; 150: 71-80.

9. Gonzalez-Angulo AM, Timms KM, Liu S, Chen H, Litton JK, Potter J et al. Incidence and outcome of BRCA mutations in unselected patients with triple receptor-negative breast cancer. Clin Cancer Res 2011; 17: 1082-1089.

10. Silver DP, Richardson AL, Eklund AC, Wang ZC, Szallasi Z, Li Q et al. Efficacy of neoadjuvant Cisplatin in triple-negative breast cancer. J Clin Oncol 2010; 28: 1145-1153.

11. Olivier M, Langerød A, Carrieri P, Bergh J, Klaar S, Eyfjord J et al. The clinical value of somatic TP53 gene mutations in 1,794 patients with breast cancer. Clin Cancer Res 2006; 12: $1157-1167$

12. Walerych D, Napoli M, Collavin L, Del Sal G. The rebel angel: mutant p53 as the driving oncogene in breast cancer. Carcinogenesis 2012; 33: 2007-2017.

13. Shibue T, Takeda K, Oda E, Tanaka H, Murasawa H, Takaoka A et al. Integral role of Noxa in p53-mediated apoptotic response. Genes Dev 2003; 17: 2233-2238.

14. Chipuk JE, Kuwana T, Bouchier-Hayes L, Droin NM, Newmeyer DD, Schuler M et al. Direct activation of Bax by p53 mediates mitochondrial membrane permeabilization and apoptosis. Science 2004; 303: 1010-1014.

15. Nakano K, Vousden KH. PUMA, a novel proapoptotic gene, is induced by p53. Mol Cell 2001; 7: 683-694.

16. Bullock AN, Fersht AR. Rescuing the function of mutant p53. Nat Rev Cancer 2001; 1: 68-76.

17. Aas T, Børresen AL, Geisler S, Smith-Sørensen B, Johnsen H, Varhaug JE et al. Specific P53 mutations are associated with de novo resistance to doxorubicin in breast cancer patients. Nat Med 1996; 2: 811-814.

18. Lim LY, Vidnovic N, Ellisen LW, Leong CO. Mutant p53 mediates survival of breast cancer cells. Br J Cancer 2009; 101: 1606-1612.

19. Vermeulen JF, van de Ven RA, Ercan $C$, van der Groep $P$, van der Wall E, Bult $P$ et al Nuclear Kaiso expression is associated with high grade and triple-negative invasive breast cancer. PLOS ONE 2012; 7: e37864. 
20. Jones J, Wang $\mathrm{H}$, Zhou J, Hardy S, Turner T, Austin D et al. Nuclear kaiso indicates aggressive prostate cancers and promotes migration and invasiveness of prostate cancer cells. Am J Pathol 2012; 181: 1836-1846.

21. Daniel JM, Reynolds AB. The catenin p120(ctn) interacts with Kaiso, a novel BTB/POZ domain zinc finger transcription factor. Mol Cell Biol 1999; 19: 3614-3623.

22. Roczniak-Ferguson $A$, Reynolds $A B$. Regulation of p120-catenin nucleocytoplasmic shuttling activity. J Cell Sci 2003; 116: 4201-4212.

23. van de Ven RA, Tenhagen M, Meuleman W, van Riel JJ, Schackmann RC, Derksen PW. Nuclear p120-catenin regulates the anoikis resistance of mouse lobular breast cancer cells through Kaiso-dependent Wnt11 expression. Dis Model Mech 2015; 8: 373-384.

24. Park JI, Kim SW, Lyons JP, Ji H, Nguyen TT, Cho K et al. Kaiso/p120-catenin and TCF/betacatenin complexes coordinately regulate canonical Wnt gene targets. Dev Cell 2005; 8 : 843-854.

25. Spring CM, Kelly KF, O'Kelly I, Graham M, Crawford HC, Daniel JM. The catenin p120ctn inhibits Kaiso-mediated transcriptional repression of the beta-catenin/TCF target gene matrilysin. Exp Cell Res 2005; 305: 253-265.

26. Bassey-Archibong BI, Kwiecien JM, Milosavljevic SB, Hallett RM, Rayner LG, Erb MJ et al. Kaiso depletion attenuates transforming growth factor- $\beta$ signaling and metastatic activity of triple-negative breast cancer cells. Oncogenesis 2016; 5: e208.

27. Pozner A, Terooatea TW, Buck-Koehntop BA. Cell-specific Kaiso (ZBTB33) regulation of cell cycle through cyclin D1 and cyclin E1. J Biol Chem 2016; 291: 24538-24550.

28. Prokhortchouk A, Sansom O, Selfridge J, Caballero IM, Salozhin S, Aithozhina D et al. Kaiso-deficient mice show resistance to intestinal cancer. Mol Cell Biol 2006; 26: 199-208.

29. Donaldson NS, Pierre CC, Anstey MI, Robinson SC, Weerawardane SM, Daniel JM. Kaiso represses the cell cycle gene cyclin D1 via sequence-specific and methyl-CpG-dependent mechanisms. PLOS ONE 2012; 7: e50398.

30. Koh DI, Yoon JH, Kim MK, An H, Kim MY, Hur MW. Kaiso is a key regulator of spleen germinal center formation by repressing Bcl6 expression in splenocytes. Biochem Biophys Res Commun 2013; 442: 177-182.

31. Koh DI, Han D, Ryu H, Choi WI, Jeon BN, Kim MK et al. KAISO, a critical regulator of p53mediated transcription of CDKN1A and apoptotic genes. Proc Natl Acad Sci USA 2014; 111: 15078-15083.

32. Yi YW, Kang HJ, Kim HJ, Kong Y, Brown ML, Bae I. Targeting mutant p53 by a SIRT1 activator YK-3-237 inhibits the proliferation of triple-negative breast cancer cells. Oncotarget 2013; 4: 984-994.

33. Punj V, Bhattacharyya S, Saint-Dic D, Vasu C, Cunningham EA, Graves J et al. Bacterial cupredoxin azurin as an inducer of apoptosis and regression in human breast cancer. Oncogene 2004; 23: 2367-2378.

34. The p53 website. Available at: http://p53.free.fr/Database/Cancer_cell_lines/Breast_cancer. html.

35. Marquette C, Nabell L. Chemotherapy-resistant metastatic breast cancer. Curr Treat Options Oncol 2012; 13: 263-275.

36. Bhattacharyya A, Ear US, Koller BH, Weichselbaum RR, Bishop DK. The breast cancer susceptibility gene BRCA1 is required for subnuclear assembly of Rad51 and survival following treatment with the DNA cross-linking agent cisplatin. J Biol Chem 2000; 275: 23899-23903.

37. Yadav BS, Sharma SC, Chanana P, Jhamb S. Systemic treatment strategies for triple-negative breast cancer. World J Clin Oncol 2014; 5: 125-133.

38. Lopes EC, Valls E, Figueroa ME, Mazur A, Meng FG, Chiosis G et al. Kaiso contributes to DNA methylation-dependent silencing of tumor suppressor genes in colon cancer cell lines. Cancer Res 2008; 68: 7258-7263.

39. Dai SD, Wang Y, Miao Y, Zhao Y, Zhang Y, Jiang GY et al. Cytoplasmic Kaiso is associated with poor prognosis in non-small cell lung cancer. BMC Cancer 2009; 9: 178

40. Cofre J, Menezes JR, Pizzatti L, Abdelhay E. Knock-down of Kaiso induces proliferation and blocks granulocytic differentiation in blast crisis of chronic myeloid leukemia. Cancer Cell Int 2012; 12: 28-2867-12-28

41. Pierre CC, Longo J, Mavor M, Milosavljevic SB, Chaudhary R, Gilbreath E et al. Kaiso overexpression promotes intestinal inflammation and potentiates intestinal tumorigenesis in Apc(Min/+) mice. Biochim Biophys Acta 2015; 1852: 1846-1855.

42. Wang H, Liu W, Black S, Turner O, Daniel JM, Dean-Colomb W et al. Kaiso, a transcriptional repressor, promotes cell migration and invasion of prostate cancer cells through regulation of miR-31 expression. Oncotarget 2016; 7: 5677-5689.

43. Liu Y, Dong QZ, Wang S, Xu HT, Miao Y, Wang L et al. Kaiso interacts with p120-catenin to regulate $\beta$-catenin expression at the transcriptional level. PLOS ONE 2014; 9: e87537.

44. Ogden SR, Wroblewski LE, Weydig C, Romero-Gallo J, O'Brien DP, Israel DA et al. p120 and Kaiso regulate Helicobacter pylori-induced expression of matrix metalloproteinase-7. Mol Biol Cell 2008; 19: 4110-4121.

45. Rodova M, Kelly KF, VanSaun M, Daniel JM, Werle MJ. Regulation of the rapsyn promoter by kaiso and delta-catenin. Mol Cell Biol 2004; 24: 7188-7196.
46. Daniel JM, Spring CM, Crawford HC, Reynolds AB, Baig A. The p120(ctn)-binding partner Kaiso is a bi-modal DNA-binding protein that recognizes both a sequence-specific consensus and methylated CpG dinucleotides. Nucleic Acids Res 2002; 30: 2911-2919.

47. Buck-Koehntop BA, Martinez-Yamout MA, Dyson HJ, Wright PE. Kaiso uses all three zinc fingers and adjacent sequence motifs for high affinity binding to sequence-specific and methyl-CpG DNA targets. FEBS Lett 2012; 586: 734-739.

48. Kim SW, Park JI, Spring CM, Sater AK, Ji H, Otchere AA et al. Non-canonical Wnt signals are modulated by the Kaiso transcriptional repressor and p120-catenin. Nat Cell Biol 2004; 6 : 1212-1220.

49. Musgrove EA, Caldon CE, Barraclough J, Stone A, Sutherland RL. Cyclin D as a therapeutic target in cancer. Nat Rev Cancer 2011; 11: 558-572.

50. Dang CV, Resar LM, Emison E, Kim S, Li Q, Prescott JE et al. Function of the c-Myc oncogenic transcription factor. Exp Cell Res 1999; 253: 63-77.

51. Bae Y-H, Shin J-M, Park H-J, Jang H-O, Bae M-K, Bae S-K. Gain-of-function mutant p53R280K mediates survival of breast cancer cells. Genes Genom 2014; 36: 171-178.

52. Braicu C, Pileczki V, Irimie A, Berindan-Neagoe I. p53siRNA therapy reduces cell proliferation, migration and induces apoptosis in triple negative breast cancer cells. $\mathrm{Mol} \mathrm{Cell}$ Biochem 2013; 381: 61-68.

53. Kim E, Deppert W. Transcriptional activities of mutant p53: when mutations are more than a loss. J Cell Biochem 2004; 93: 878-886.

54. Neve RM, Chin K, Fridlyand J, Yeh J, Baehner FL, Fevr T et al. A collection of breast cance cell lines for the study of functionally distinct cancer subtypes. Cancer Cell 2006; 10: 515-527.

55. Daniel JM, Ireton RC, Reynolds AB. Monoclonal antibodies to Kaiso: a novel transcription factor and p120ctn-binding protein. Hybridoma 2001; 20: 159-166.

56. Wu J, Mariner DJ, Thoreson MA, Reynolds AB. Production and characterization of monoclonal antibodies to the catenin p120ctn. Hybridoma 1998; 17: 175-183.

57. Kao KJ, Chang KM, Hsu HC, Huang AT. Correlation of microarray-based breast cancer molecular subtypes and clinical outcomes: implications for treatment optimization. BMC Cancer 2011; 11: 143.

58. Desmedt C, Di Leo A, de Azambuja E, Larsimont D, Haibe-Kains B, Selleslags J et al. Multifactorial approach to predicting resistance to anthracyclines. J Clin Oncol 2011; 29: 1578-1586.

59. Sabatier R, Finetti P, Cervera N, Lambaudie E, Esterni B, Mamessier E et al. A gene expression signature identifies two prognostic subgroups of basal breast cancer. Breast Cancer Res Treat 2011; 126: 407-420.

60. Sabatier R, Finetti P, Adelaide J, Guille A, Borg JP, Chaffanet M et al. Down-regulation of ECRG4, a candidate tumor suppressor gene, in human breast cancer. PLOS ONE 2011; 6 : e27656.

61. Li Y, Zou L, Li Q, Haibe-Kains B, Tian R, Desmedt C et al. Amplification of LAPTM4B and YWHAZ contributes to chemotherapy resistance and recurrence of breast cancer. Nat Med 2010; 16: 214-218.

62. Loi S, Haibe-Kains B, Majjaj S, Lallemand F, Durbecq V, Larsimont D et al. PIK3CA mutations associated with gene signature of low mTORC1 signaling and better outcomes in estrogen receptor-positive breast cancer. Proc Natl Acad Sci USA 2010; 107 10208-10213.

63. McCall MN, Bolstad BM, Irizarry RA. Frozen robust multiarray analysis (fRMA). Biostatistics 2010; 11: 242-253.

64. Benito M, Parker J, Du Q, Wu J, Xiang D, Perou CM et al. Adjustment of systematic microarray data biases. Bioinformatics 2004; 20: 105-114.

65. Team RC. A language and environment for statistical computing. Austria R Foundation for Statistical Computing: Vienna, 2014.

Cell Death and Disease is an open-access journal published by Nature Publishing Group. This work is licensed under a Creative Commons Attribution 4.0 International License. The images or other third party material in this article are included in the article's Creative Commons license, unless indicated otherwise in the credit line; if the material is not included under the Creative Commons license, users will need to obtain permission from the license holder to reproduce the material. To view a copy of this license, visit http://creativecommons.org/licenses/by/4.0/

(C) The Author(s) 2017

Supplementary Information accompanies this paper on Cell Death and Disease website (http://www.nature.com/cddis). 